\title{
Describing a Craniofacial Anomaly: Finite Elements and the Biometrics of Landmark Locations
}

\author{
FRED L. BOOKSTEIN \\ Center for Human Growth and Development, The University of Michigan, \\ Ann Arbor, Michigan 48109
}

\begin{abstract}
KEY WORDS Tensor biometrics, Statistics of shape, Apert
\end{abstract} syndrome, Deformation, Morphometrics

\begin{abstract}
An intergroup comparison of cephalometric landmark configurations by the finite-element method elegantly depicts the algebra of some of the size and shape change measures that one may define by reference to those landmarks. In studies of mean differences between groups, the statistical analysis of these finite elements is equivalent to competent statistical analysis of the same data using any other geometric metaphor, such as properly standardized vector descriptions of landmark "movement" or scalar measures, size and shape variables, taken in sufficient variety. In applications to landmark data, the reality of finite-element depictions is purely statistical rather than phenomenological. In the absence of additional evidence, they should not be held either more or less biologically meaningful than other descriptions of the same landmark changes to which they lead. These propositions are exemplified using landmark data from 13 cases of Apert syndrome.
\end{abstract}

In several recent articles, the method of finite-element analysis has been applied to data in the form of cephalometric landmark locations. Some of these presentations use triangles (Moss et al., 1985), others quadrangles (Cheverud et al., 1983); mine use both triangles and curvilinear polygons (Bookstein, 1978, 1984a,b, 1986). The statistical counterpart of all these efforts is the tensor biometrics under development by myself, Colin Goodall (1983), and others. Together these techniques, one new, the other newly applied, attempt to turn D'Arcy Thompson's notion of Cartesian transformation into a workable method for routinely describing and testing differences in growth and form.

The two approaches analyze the same data bases of digitized landmark coordinates and draw the same diagrams of triangles, circles, ellipses, and crosses; but they have begun to diverge in their vocabularies of interpretation. Whereas the finite-element literature usually speaks about real deformation (changes of material shape in between landmarks, in small regions as well as large), tensor biometrics deals with size and shape variables referring only to the landmark locations available for analysis, not the regions around or between them. Each variable expresses a particular trend, change, or comparison to a greater or lesser extent.
In this comment I argue that as currently applied in cephalometrics the finite-element method reports relationships among alternate measurement schemes rather than any underlying material phenomenon. Different finite-element schemes may not be distinguishable by statistical criteria; nor is it necessary that a data analysis carried out by the machinery of finite deformation analysis be reported in those terms. The statistics of a sufficiently diverse $\operatorname{set}^{1}$ of conventional variables-distances between landmarks, angles and ratios, or "vectors" of landmark displacement in an appropriately registered coordinate system-are indistinguishable from the statistics of finite elements based on landmark locations whenever both statistical analyses are carried out correctly. The biological interpretation of a cephalometric data set must be the interpretation of that common multivariate core of statistical sig. nal, not of whatever accidentally measured "variables," or even "finite elements," particular investigators proceeded with in their computations.

\footnotetext{
${ }^{1}$ The measures recorded must constitute an archive of the landmark configuration: they must contain all the information necessary to reconstruct their relative positions in full detail.
}

Received April 25, 1986; revision accepted June 3, 1987 
No two fair summaries of the same Cartesian coordinate data can contradict each other: geometry is geometry. But in practice, whenever two biometers are beholden to different particular sets of measurements, the one cephalometric analysis can be made to appear contradicted by the other. The strength of tensor analysis lies in the disentangling of these interpretive difficulties. Because good biological interpretation is univariate, whereas the statistics of form is multivariate, the geometric justification of a choice of interpretation matters a great deal for the quality of biological insight. I argue below that one version of the finite-element analysis suits the multivariate statistics of form very well and permits the most appropriate (i.e., informative) cephalometric description to be deduced highly objectively and reliably. But neither tensor analysis nor any other analysis of landmark data may be used, for instance, to determine "where the problem originates"-for this is not a multivariate question, but a biological one.

Craniofacial anomalies are distributed. Almost everywhere across the affected head, nearly all measurements, conventional or tensor, will tend to be abnormal. In this apparent richness of descriptive material, some features will be far more abnormal than others. But there are strong constraints on the distribution of these characteristically most anomalous variables, constraints owing purely to the geometric origin of alternate measurements. Indeed, it is easy to show that in any anomaly some features must be normal, precisely (Bookstein, 1983). This is a mere fact of geometry, having no biological content, not even the notion of "normal." But whereas biology is the discussion of syndromes, geometry is merely the discussion of rulers and protractors. If one does not understand when the values of two alternate measurements are expressing a contingent relationship, and when instead a necessary one, then one cannot tell the difference between biology and geometry in one's findings.

The foundation of morphometrics is at the same time the crux of its clinical relevance: it is the understanding of ways in which geometry constrains biological or clinical insight. Only via this understanding can we ultimately combine disparate and distributed findings in a rational way. One sensible set of variables, for instance, incorporates a large variety of distance-measures, such as a conscientious clinician might record by ruler or dividers. It then becomes the morphometrician's task to indicate how the distances (or angles, or ratios) that are most informative, syndrome by syndrome, should be combined and balanced in a report. A somewhat more effective approach is via the consideration of all possible measures at the same time for simple sets of landmarks, such as triangles; this is tensor biometrics.

The tensor description ultimately results in a prescription of appropriate measures for characterizing an anomaly: those measures most abnormal, together with those least abnormal, triangle by triangle. When shape variables are generated as ratios of distances at $90^{\circ}$, then these pairs of most-and-least are associated with pairs of shape variables at $45^{\circ}$ (Bookstein, 1983). It is reasonable next to inquire how such descriptions interact among different triangles. How does one combine alternate local descriptions of shape change into a most meaningful overall scheme? The purpose of this paper is to explore, in a preliminary way, the aspects of description of anomaly which are common to diverse triangulations of a set of landmarks. These are, by theorem (Bookstein, 1986), the statistical underlayment of the biological phenomenon the clinician is pursuing.

\section{REPORTS OF SHAPE CHANGE}

Case by case or average by average, one can report information about a shape change systematically whether one talks about scalars (numbers), vectors (arrows), or tensors (crosses). Figure 1 shows how the same change in relative positions of three landmarks, frame a, can be expressed equally effectively by way of a variety of geometric metaphors. Figure 1b draws the change from left to right as a deformation of the interior of a triangle, after the fashion of D'Arcy Thompson. In frame $c$ this deformation is reexpressed as a conjoint change in all distances measurable across this triangle of landmarks. The continuum of distance-measures is redundant; the pattern of their changes can be summarized, as in frame d, by the perpendicular pair of principal directions characterized as having greatest and least strain, or rate of change of distance. In this example, one length has increased by $10 \%$, and the other has decreased by $9 \%$.

The style of report in frame $\mathbf{d}$ is a tensor presentation independent of any specification of a coordinate system. However, there are many other coordinate-independent 


\section{$\times$}

(a) +

(b)

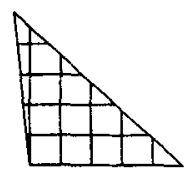

$x$

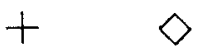

(d)

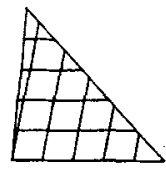

(c)
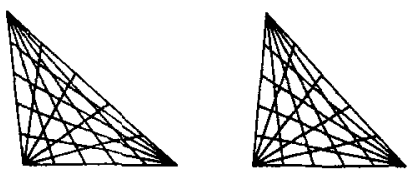

(e)

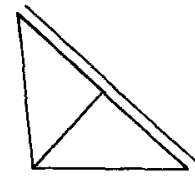

h)

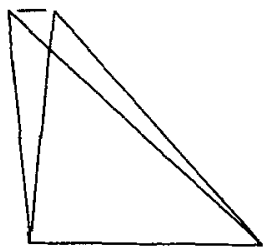

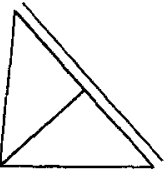

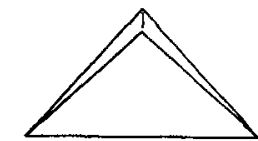

(g)

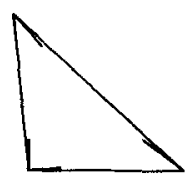

Fig. 1. Various cephalometric languages for the change in a configuration of three landmarks drawn in panel a. b: Cartesian deformation, after D'Arcy Thompson. $\mathbf{c}$ : Changes in length of a continuum of distancemeasures crossing the form. d: Tensor analysis: principal directions starting and finishing at $90^{\circ}$ and bearing greatest and least rates of change of length, or principal strains. e: Lengths along the principal directions as variables measured along transects of the form. The ratio between the indicated distances is the shape variable most sensitive to this deformation. $f$ : Changes in lengths of three sides. g: Changes in three angles (schematic). $\mathbf{h}$ : After correcting for the change in separation of any two landmarks, the third landmark may be considered as having "moved" with respect to a baseline fixed upon the other two. After information about size change is restored to versions $g$ and $h$, an appropriate multivariate statistical analysis of groups differing or changing in this way results in the same algebraic findings regardless of the representation of shape change that is used. modes of reporting the same change. For instance, one might exploit the language of scalar variables rather than tensors but use the tensor analysis to select a single most appropriate variable. For the change shown in Figure 1, one most sensitive shape variable is the ratio of distances measured parallel to the cross of principal directions and, for convenience, passing through at least one landmark each, as in frame e. This ratio 

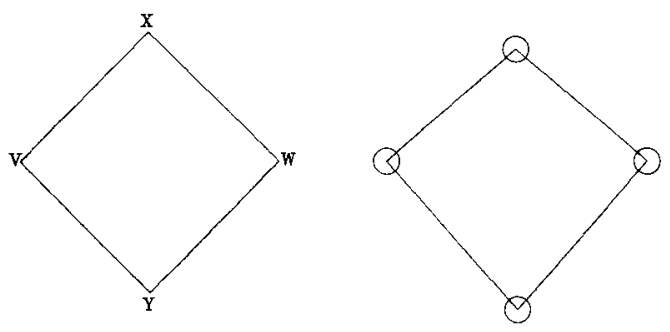

Fig. 2. Design of a simulation for a transition from square to kite shape. All forms begin exactly square on a 20-unit diagonal and "grow" into configurations which vary from the kite shape by independent normal deviations of variance 1 unit in every direction at each of the four landmarks. The net shape change (asymmetry of the kite about the horizontal) has been exaggerated almost threefold for legibility; the t-ratios reported in the text and in the legends of Figures 3 and 4 correspond to a much subtler change-for instance, half the amount of shape change shown here together with a sample sized 25 instead of 50 .

changes from 0.45 to 0.55 over the transformation. Other scalars may satisfactorily report this change in sets of two or three. Frame $f$ reports the change as changes in the sides of triangle by fractions $0.0,0.0,-0.095$; frame $\mathrm{g}$, as increments of $-11.4^{\circ}, 5.7^{\circ}$, and $5.7^{\circ}$ in the angles of the triangle. Finally, frame $h$ shows three equally correct vector interpretations: the relative motion of any one of the landmarks with respect to two others after correction for the change in the distance between those two. (This correction for change in baseline distance overcomes the only serious objection to conventional systems of registration and orientation in cephalometrics; cf. Bookstein, 1984b.)

The formal geometry of frames $c-h$, common to both finite-element analysis and tensor biometrics, makes no reference to any information beyond the locations of landmarks. The edges of the triangle are abstractions, the interior likewise. In this context, all lines in frames $\mathrm{c}$ and $\mathrm{e}$ must be taken to depict point-to-point length measurements rather than straight-line loci cutting across tissue. What the geometry of this mathematical triangle supports, and the tensors depict, is in fact the algebraic structure of these distance variables and of the shape variables derived from them (Bookstein, 1986).

Finite-element analysis was developed to exactly suit the phenomena of continuum mechanics: to express real stress-strain relations and conservation laws in material sys- tems. But the deformation model of D'Arcy Thompson is an abstract transformation, unfounded as biomechanics whenever there is heterogeneity of material content inside. This is certainly the case for polygons of cephalometric landmarks about the face. The aspect of cephalometrics which smooths out the complicated internal structures into a continuum is the application of a ruler or protractor, that is, the operation of measurement. In the application of finite elements to cephalometrics, then, the appropriate analogy is not of craniofacial process with continuum mechanics, but of landmark-based measurements with continuum mechanics. The areas between landmarks supply no information not already available from landmark locations alone. From data so sparse there is no best way, only several equivalently thorough ways, equivalent geometrical metaphors, to report a form-change or a pattern of influence upon form-change. (There is an extended discussion of alternate measurement schemes in Bookstein et al., 1985.) The formalism of finite elements, without any necessary relation to the biological phenomenon under study, nevertheless exactly suits the task of coordinating all these biometrical reports. That is, tensors computed from landmark data depict the measurement of craniofacial form-change, not the phenomenon.

\section{SQUARE TO KITE: THE EQUTVALENCE OF ALTERNATE CEPHALOMETRIC METHODS}

I will demonstrate this radical biometric relativism by studying one shape change according to diverse biometrical methods and showing their findings to be identical. The example is artifical, exploiting some unrealistic symmetries so as to ease verbal presentation of the equivalents.

Consider the shape change shown in Figure 2 , taking an originally square configuration of landmarks VXWY into the form of a kite shown at the right. Around this particular change there is assumed to be individual variation in the final positions of the landmarks, variation which is distributed independently from landmark to landmark according to a circular normal pattern (Bookstein, 1984a, 1986) as sketched by the circles about the landmark locations in the right-hand panel, the "later" locations. Suppose the available sample size is 50 . Let the original square of landmarks have a diagonal 20 units long; let the amount of shape 


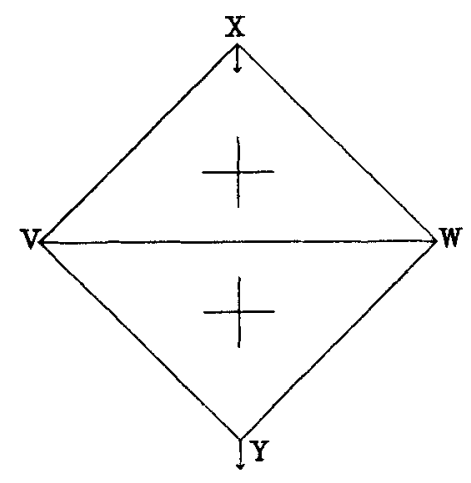

Fig. 3. Biometrical analysis of the change in Figure 2 according to one finite-element scheme. Each change of triangular shape is displayed as the displacement of one landmark with respect to the two landmarks of the baseline both fixed in position (cf. Fig. 1h.) The shape changes of the two triangles are uncorrelated under the assumptions of Figure 2. The shape variables most sensitive to these changes, constructed as empirical distance-ratios, are indicated triangle by triangle. The combination of these two into one best four-landmark shape score is the ratio of heights of the triangles above their common baseline, with matched t-statistic 3.5 for the change. (See the text for interpretation of this $t$.)

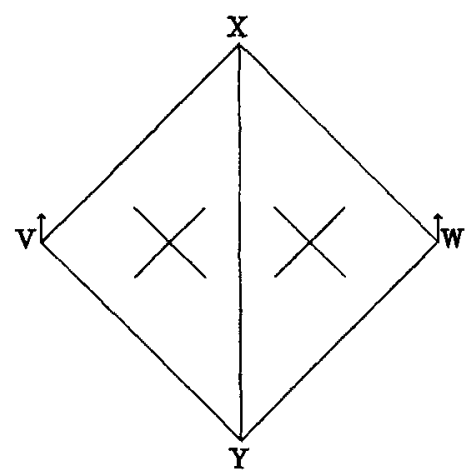

Fig. 4. The same change according to an alternate triangulation. The best shape variables for the separate triangles may be taken as the ratios of side indicated by the crosses; the best combined measure is now algebraically equivalent to the ratio of the summed upper two edges of the quadrilateral to the summed lower two edges. The matched $t$ is again 3.5 .

change be calibrated by a change equal to 0.5 unit in the relative location of its diagonals (a change exaggerated to 1.4 units in the diagram for legibility); and let the standard deviations of landmark locations about their mean positions be 1 unit in each direction, landmark by landmark, as indicated by the radii of the circles.
Notice that this transformation is in the language of Bookstein (1985), purely inhomogeneous: the diagonals of the quadrilateral are unchanged from those of the square in both length and orientation. By virtue of this double invariance, the tensors for the separate triangles in either triangulation to follow "sum" to a net homogeneous component of zero, that is, no change-not a helpful point of view at all.

The biometrical exercise this example is intended to support is twofold: the description of the mean shape change from the square to the kite, together with the testing of that change for statistical significance. The statistical analysis will involve matched $t$ tests for changes from the fixed starting form. For each shape measure that we come to consider, I will cite without proof its mean change from left to right and its standard deviation in the forms at the right owing to the circular landmark noise we assumed. These quantities may be inferred from the setup in Figure 2 by algebra which will not be reproduced here (cf. Bookstein, 1986). For each measure, $\sqrt{ } 50$ times the ratio of its mean change to the standard deviation of that change serves approximately as the square root of an F-ratio for testing the significance of shape change from the starting square form. In general, this $\sqrt{ } \mathrm{F}$ has four degrees of freedom for the numerator. To be thought of as an approximate t-test, it must be deflated by about $20 \%$ if we stumbled into the shape variable under consideration from the class of all possible shape measures of a triangle, and by about $38 \%$ if we stumbled into it for describing the change of a quadrilateral; see Bookstein (1984a). In the interest of simplicity, I will assume instead that we suspected kite-shaped-ness all along, so that the ratio of mean change to its own standard error can be taken at face value as a t-statistic.

Let us begin by considering the simple but arbitrary finite-element scheme shown in Figure 3. Registering on the landmarks of the common baseline (Bookstein, 1984b), we find the mean shape change of each triangle from left to right to be represented by the vectors shown. (These are really pseudovectors, Bookstein 1984b, as they behave differently than vectors do when we change coordinate systems.) The shape variables capturing these changes may be taken as the ratios of size variables corresponding to the tensor crosses inside the triangles. In either triangle, the ratio most sensitive to this particular shape change is the aspect ratio of the 

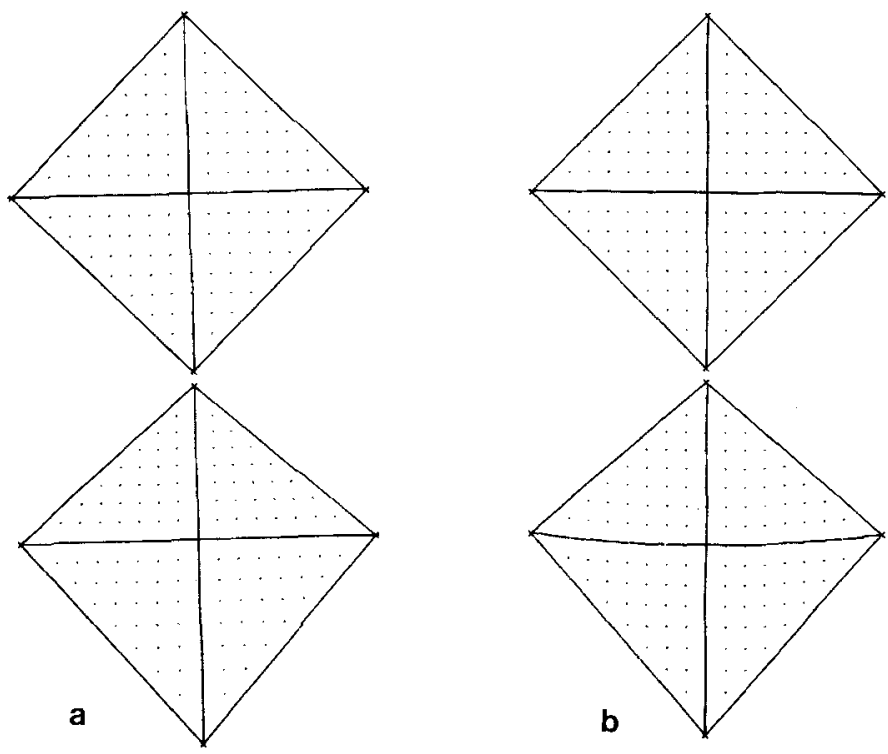

Fig. 5. The same change, according to two versions of a quadrilateral element. a: Projection, leaving both diagonals straight but nonlinear everywhere. b: Bilinear transformation, linear on all four edges of the square, bending one diagonal and mildly nonlinear along the other.

triangle, its height divided by the length of its base. From the numerical specifications of the change (recall that the diagrams exaggerate this change threefold) one can infer that the net shape change of either triangle is represented by a change of .025 in the mean of this ratio, which has, furthermore, a population standard deviation of .07 . Then the "t-ratio" for testing the change from the starting right triangle will be $.025 /(.07 / \sqrt{ } 50)$ $\sim 2.5$, which is significant at the $1 \%$ level (if we had intended all along to ask about this particular ratio) or at about the 5\% level (if we stumbled into this ratio as the empirically strongest index of change from left to right).

The shape change from square to kite is equally pronounced in both triangles of the figure. In the upper triangle the aspect ratio has decreased by .025; in the other, it has increased by the same amount. By virtue of the example's symmetry, the morphometric variable most sensitive to the effect of this simulated shape change is constructed by cancelling the common denominator of the two ratios, the diagonal of the triangulation, so as to arrive at the ratio of the two heights of the two triangles. This ratio is better taken in the form of the ratio of one height to their sum. The mean change of this proportion in the simulated sample is again .025 , the standard deviation .05 , and so the appropriate test statistic is $.025 /(.05 / \sqrt{50})=3.5$. This is sharper by a factor of $\sqrt{ } 2$ than the ratios for the shape changes in the triangles separately because it is the sum of two independent, equally precise estimates of the same phenomenon.

Consider, now, the triangulation in Figure 4 , based upon the other diagonal of the square. The effect of this shape change on either of these triangles is quite different from that in Figure 3. For these triangles, the change is equivalent to a shear of the third landmark parallel to the baseline, and corresponds (Bookstein, 1983) to principal axes of shape change that are at $\pm 45^{\circ}$ to the baseline, so that they lie along the sides of the square, triangle by triangle, as shown. Segments VY and WY grow by $2.5 \%$, while VX and WX shrink by the same fraction. The shape variable best expressing this change may be taken as the ratio VX/VY for the lefthand triangle, WX/WY for the right. The mean change of either ratio from the "earlier" square to the "later" form is .05 , the standard deviation .14.

As for the other triangulation, the most sensitive four-landmark variable for describing and testing the tendency to kite-shaped- 
ness simply adds together the two separately optimal shape variables for the triangles separately. Because of the symmetry of the design, this is equivalent to summing the two numerators, then dividing by the sum of the two denominators, to arrive at the ratio $(\mathrm{VX}+\mathrm{WX}) /(\mathrm{VY}+\mathrm{WY})$, the sum of the lengths of the upper two edges divided by the sum of the lower two. As before, to improve the statistical behavior of the ratio we replace the denominator by the sum of numerator and denominator, the sum $\mathrm{VX}+\mathrm{VY}+\mathrm{WX}+\mathrm{WY}$ of all four sides involved. In this form, the fraction has a mean change of .0125 from square to kite, with a standard deviation of .025 . The t-ratio corresponding to the observed change in this fraction is thus .0125 / $(.025 / \sqrt{ } 50)=3.5$, just the same as for the ratio of heights in the first triangulation. The correlation of these two shape variables, one based on one triangulation, one on the other, is .992-they are measuring precisely the same geometric phenomenon, up to small nonlinearities remaining in the algebra. Hence the biometrics of this change is independent of triangulation.

Other attempts to derive a shape measure customized for this particular shape change will arrive yet again at the same end result, essentially the same variable. For example, noticing that the effect of the change is to move the diagonal XY along the diagonal VW without rotation or change of length, one might construct a third clever shape measure which is the proportion in which diagonal XY cuts diagonal VW. This proportion will be found to have sample standard deviation twice its mean change, 0.025 , from square to kite, so that it encapsulates the observed difference of form with the same precision as the other two indices, and indeed is correlated with them by the same coefficient, .992: it is just another version of the ratio of altitudes already discussed in connection with Figure 3. Or one might consider the difference between the angles of the quadrilateral measured at $\mathrm{X}$ and at $\mathrm{Y}$; over the shape change, this difference changes by an average of $5.7^{\circ}$ with standard deviation $11.4^{\circ}$, leading, once again, to a t-ratio of 3.5 and nearly perfect correlation with the other descriptive measures.

This statistical equivalence notwithstanding, the different geometric versions of changes interior to the landmarks are flatly incompatible. For instance, the first triangulation and the second blatantly contradict each other. That of Figure 3 leaves line VW straight; that of Figure 4 breaks it into a pair of oblique segments. The transformation of Figure 4 models the change of diagonal XY as linear, while that of Figure 3 presumes it highly nonlinear, growing at the bottom but shrinking at the top. A composite effect on the "interior" might be modeled D'Arcy Thompson-style as the projection mapping in Figure 5a, which leaves straight lines straight but is nonlinear everywhere. Or we might have chosen instead the bilinear mapping of Figure 5b. This map (cf. Bookstein, 1985) is linear on all four external edges of the quadrilateral of landmarks but bends one diagonal and transforms the other nonlinearly. Neither of these global finite elements is consistent with either triangulation. There is no reason to expect any of these maps to relate accurately to biological reality inside this quadrilateral of landmarks in the cephalogram or in the head. Finite-element models applied to landmark data deal with the algebra of alternate measurement schemes, not biological reality.

\section{GENERALITY OF THESE FINDINGS}

The equivalence just exemplified is always the case, though the general development is algebraically complicated (Bookstein, 1986). Shape variables synthesized so as to be optimally sensitive to a mean change or other biometric effect upon a set of landmarks will be statistically indistinguishable regardless of whether a finite-element analysis has been used and, whenever one is used, regardless of which finite-element decomposition is involved. There is no analogue to this ambiguity in other applications of finite-element modeling, where the governing principles are the constitutive laws of continuum mechanics instead of the biological rules of homologies between points across populations.

Which of the geometric metaphors of change in Figures 3-5 are supported by the available data, the coordinates of those four landmarks? Clearly, none of them. The coordinate data determine the biometric specification and testing of an effect, but do not tell us how to depict the mechanism of that effect. That mechanism is located in the region between landmarks, where there are no data. When the information available is limited to locations of homologous landmarks, a variable found to be most sensitive by analysis of all the distances between pairs of landmarks and constructed landmarks will be correlated nearly perfectly with the quite different-looking variable found to be most sensitive by 
14 VS 7
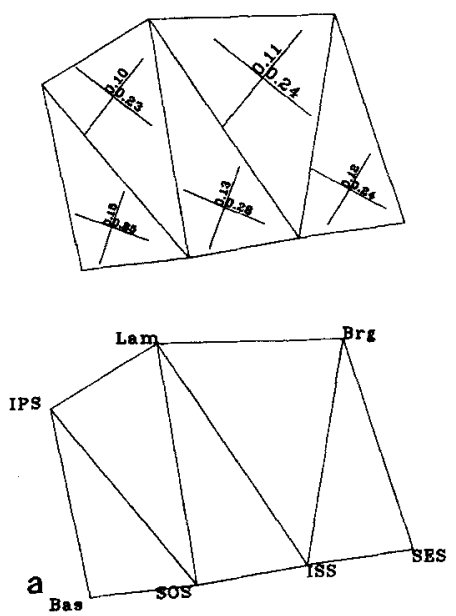

14 VS 7
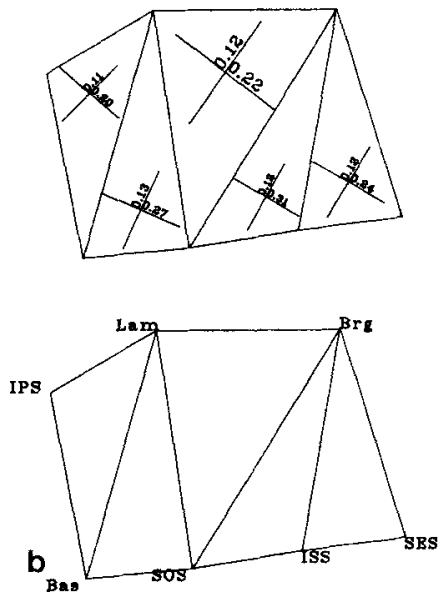

7 DA

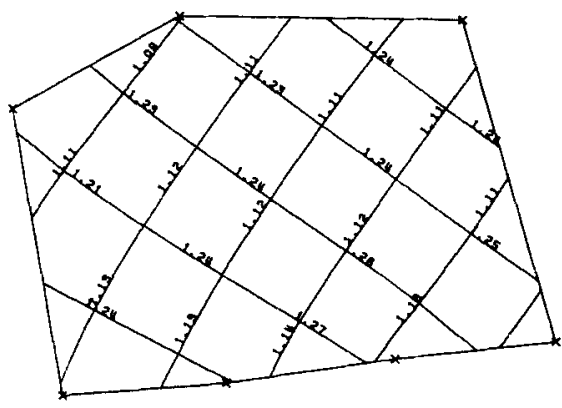

C

$14 D A$

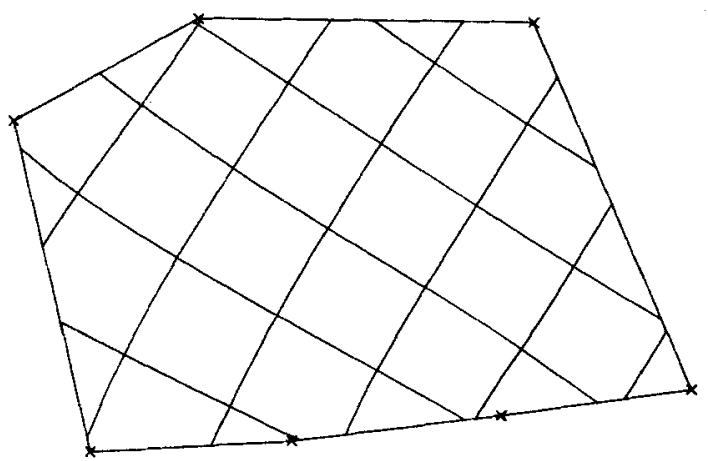

Fig. 6. Three finite element schemes for growth of the neural skulls of 21 purebred rats from 7 days of age to 14 (data courtesy of M. Moss and H. Vilmann). Landmarks: Bas, basion: SOS, spheno-occipital synchondrosis; ISS, intersphenoidal synchondrosis; SES,

finite-element analysis, biorthogonal analy. sis, or the analysis of relative "movement" of landmarks to a common arbitrary baseline. Whether one chooses local or global elements, triangles, quadrilaterals, or bior- sphenoethmoid synchondrosis; Brg, bregma; Lam, lambda; IPS, intraparietal suture. a,b: Two triangulations in substantial agreement. c: The biorthogonal grid, a global finite element smoothing over alternatives like $a$ and $b$.

thogonal grids, the finite-element analysis is a helpful picture of this common biometrical core.

For a data base consisting of point-coordinates, there is at root only one multivariate 


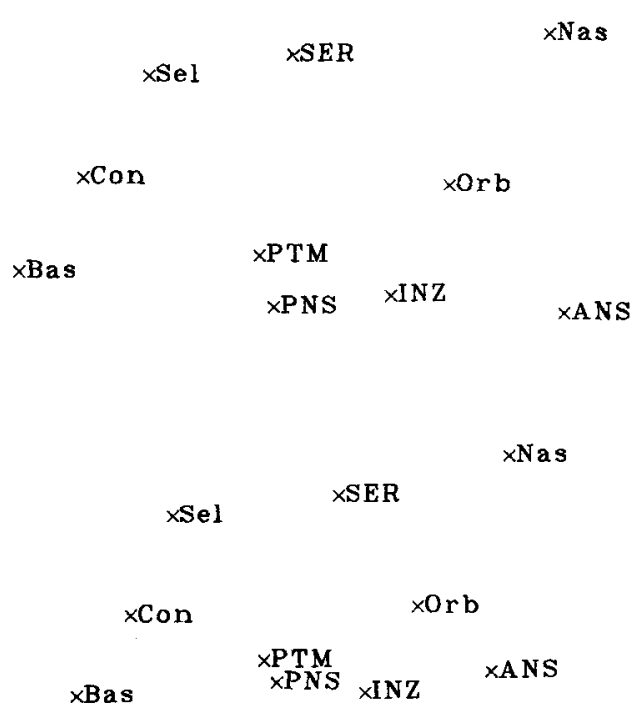

Fig. 7. Mean positions (to the Frankfurt horizontal) of ten landmarks from the lateral cephalogram in a sample of Apert cases (lower set) vs. their positions in an age and sex-matched normative sample of Ann Arbor children from the 1960s (upper set). These landmarks will be used in various unconvincing finite-element analyses of Apert syndrome as a deformation (Fig. 8) and will be summarized in the scheme of Figure 9 , which does not refer to deformations.

statistical analysis of size and shape (Bookstein, 1986). But these analyses reside in a space of $2 k-3$ dimensions (where $k$ is the number of landmark points involved), not in the two-dimensional space of the data. The diagrams of tensor analysis reexpress these findings in the more familiar space of the cephalogram, a space which must not be confused with the real spaces inside the head.

Demonstrably homogeneous transformation: The gap between the two senses of the single term "deformation"-one geometrical, one biological-should be bridged only in the presence of explicit evidence that the implied deformation is independent of the particular finite-element scheme used to describe it. ${ }^{2}$ Figure 6, for instance, involves seven landmarks located on lateral cephalograms of the rat neurocranium. The elements are summarizing the shape change of these land-

\footnotetext{
${ }^{2}$ This requirement is acknowledged whenever finite-element methods are applied in continuum mechanics, the field for which they were developed.
}

marks in 21 rats followed from 7 days of age to 14 . Analyses of these data, originally collected by Henning Vilmann, have been published several times (Bookstein, 1983; Patel, 1983; Moss et al., 1985). Each analysis invokes its own finite-element decomposition, but they all agree about the phenomenon under consideration. The strains modeled here appear to change hardly at all when we pass from the triangulation of Figure $6 \mathrm{a}$ to the "opposite" triangulation of Figure 6b, or combine these and all other potential elementary schemes in the global finite element (biorthogonal grid) of Figure 6c.

In Bookstein and Sampson (submitted for publication) it is shown that the growth of the rat calvarium over these 7 days may be described satisfactorily by a homogeneous component that explains $91 \%$ of its effect upon the landmarks severally. The principal strains of this homogeneous component lie along the average directions of the grid curves in Figure 6c. (The other 9\% of the change observed is mostly a deficit of longitudinal growth rate along the top of the skull, intraparietal suture (IPS)-bregma, relative to the growth rate observed along the bottom, basion-spheno-ethmoid synchondrosis (SES).) In this example, the empirical nearhomogeneity of observed "growth strain" is expressing a nearly homogeneous biological process underlying the strain: the continuing directional growth of the normal rat brain. For further interpretation of this phenomenon, see Moss et al. (1985).

\section{THE CALVARIAL DEFORMITY IN APERT SYNDROME}

In order to relate these cautions to the topic of this symposium, the analysis of craniofacial dysmorphology, I shall reanalyze some cephalometric data from 13 patients with Apert syndrome. The data were kindly sent to me by Dr. Joseph MeCarthy from the patient files he maintains at the Institute for Reconstructive Plastic Surgery, New York University. Some of these findings have been published in Bookstein (1984b) and in Grayson et al. (1985). Apert syndrome, along with Crouzon syndrome, is a cranial synostosis characterized by premature closure of the intracranial bony sutures about the maxilla and frontal bone. The genetically distinctive version which is Apert syndrome, or acrocephalosyndactyly, shows deformities of the extremities as well. Facially, the syndromes typically include a high, bulging forehead 

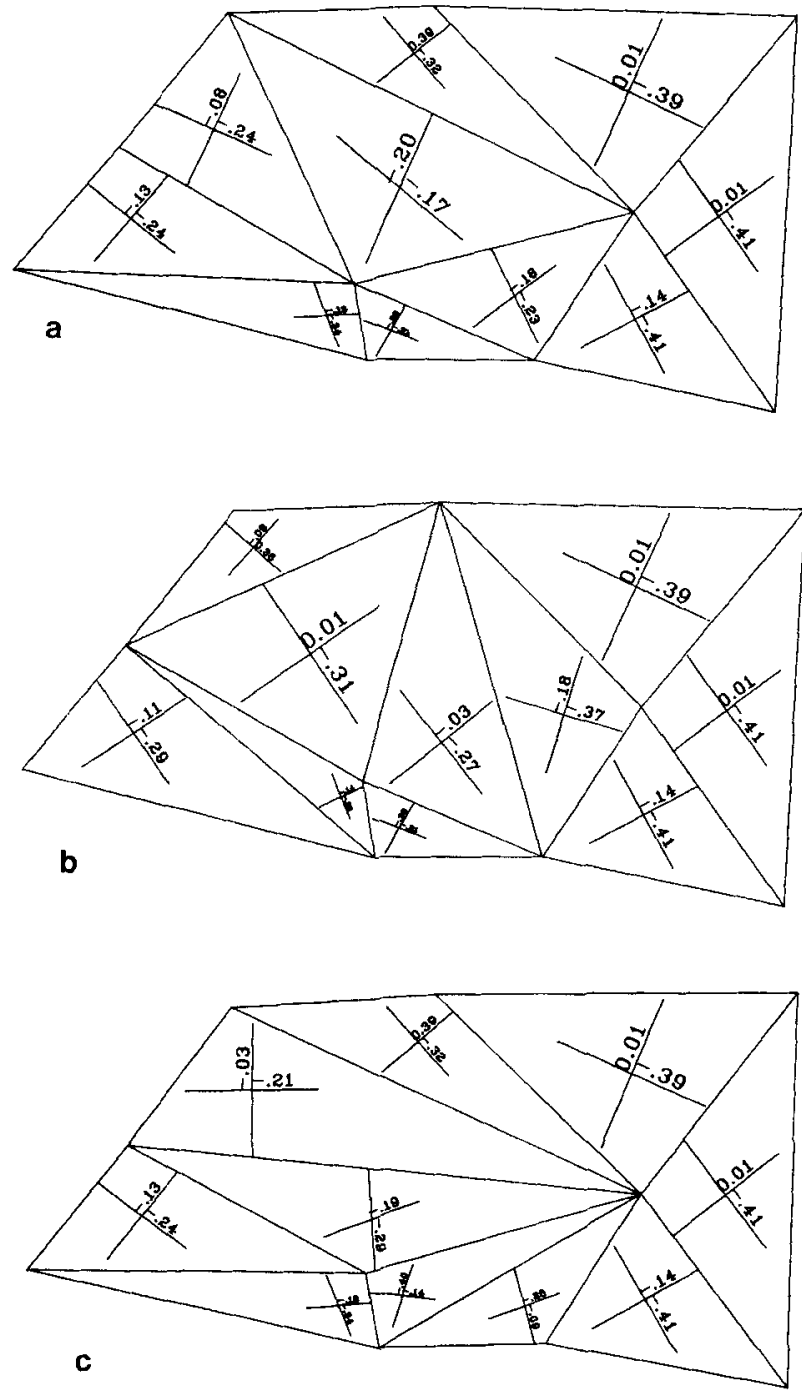

Fig. 8. Attempts to describe the calvarial deformity as deformation in 13 Apert cases. These mean tensors are computed by the method of Bookstein (1982) and tested according to the approximations of Bookstein (1984a). For each triangle, 60 size variables are considered, distances from each landmark to 20 points evenly dividing the side opposite. Each of these size variables has an empirically observable strain from normative mean form to each Apert case. For each triangle, the average deformation is not computed as a matrix, but, rather, is directly characterized by the size variables which bear empirically greatest and smallest mean ratios of deformity (Apert length divided by mean normative length) averaged over the 13 cases. On the model of homogeneous deformation, these strains apply to every distance measure to which they are parallel; I draw them, by convention, through the center of the circle inscribed in the mean form of the element, with length equal to the radius of that circle. The difference between these mean ratios, divided by the sum of the sample standard errors of the ratios, may be used as a test statistic for significance of shape change in that triangle; it is proportional to an approximate t-ratio and has a $5 \%$ significance level of about $\sqrt{ } \pi \sim 1.77$. Axes not nearly at $90^{\circ}$ correspond in practice to a finding of insignificance for this component of the change. More rigorous procedures for the analysis of data sets like these, suitable for larger samples, are reviewed in Bookstein (1986). a-c: Mean tensors for triangles in three different tesselations of the configuration of ten landmarks, expressing three mutually incompatible finite deformations. Each of these models expresses the same biometric analysis, and therefore none of them can be considered a picture of true biological deformation. Their truth is limited to the space of alternate shape and size measures. d: Additional triangles in the cranial base. The deformations implied by these triangles contradict schemes a-c. 

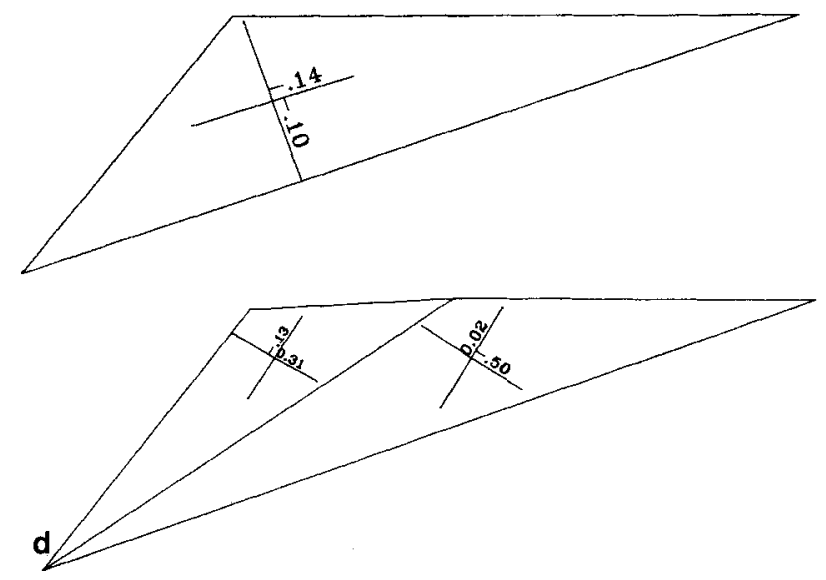

Fig. 8. Continued.

and a short maxilla positioned further back than normal.

Beginning with the 40-odd landmarks customarily available on the lateral cephalogram, I excluded those on the dentition. Because of the open-bite usual in Apert cases, I have further restricted my attention to the calvarium. There remain the ten landmarks indicated in Figure 7. The conventional cranial base is delimited by sella, basion, and nasion. A fourth "cranial base" landmark, sphenoethmoid registration point (SER), is a useful artifact of the lateral projection; it is located at the intersection of the shadows of the greater wing and the body of the sphenoid. Three landmarks lie laterally on the maxilla broadly construed: inferior zygoma (INZ), orbitale, and pterygomaxillary fissure (PTM). The landmark condylion, though technically on the mandible, is used here to indicate a location at the temporomandibular joint upon the temporal bone. Finally, two landmarks lie on the midline of the maxilla: anterior nasal spine (ANS) and posterior nasal spine (PNS).

The statistical comparisons to follow construe the deformity of the Apert case as the deformation into his or her form from the age- and sex-matched University of Michigan University School Study normative mean landmark locations (Riolo et al., 1974). By theorem 2 of Bookstein (1986), any size variable whose mean value in the Apert cases shows an extreme of contrast with respect to the normal mean can be defined using at most three landmarks. Hence we survey the biometrics of the syndrome quite efficiently by computing mean shape changes for a va- riety of triangular parcellations of the form, as in Figure 8a-d, and subsequently interpret these mean differences in terms of size and shape variables measured across the form. The caption to this figure sketches the algorithm by which these means were computed and tested; details of these steps are to be found in Bookstein $(1982,1984 a)$.

For instance, the most reduced maxillary distance (in this lateral projection) is the separation between orbitale and ANS, as seen in either of the triangles involving ANS at lower right in Figure 8a-c. This distance falls short of normal by some $41 \pm 10 \%$ (S.D.). In the cranial base, one constructed distance (Fig. 8d) has shrunk by a greater fraction: the distance from SER to a point $75 \%$ of the way from basion to nasion has decreased from the normal mean by $50 \pm 21 \%$. Contrariwise, certain aspects of the mean Apert form differ from the normal mean hardly at all. For example, the cranial base triangle basion-sellanasion, although it falls about $12 \%$ short of the matched normative mean form in general size, is almost identical with normal in mean shape--there is only a $4 \%$ mean disproportion, best measured as a change in the cranial base angle.

But these statistical summaries notwithstanding, nothing in the "deformation" bearing the sample statistics comparing Aperts to normals bears automatic interpretation as biologically real deformation somehow typical of the patients' heads. Instead, we find ourselves in the situation of Figures 3-5: different triangulations imply utterly different deformations that cannot be statistically resolved. 
Consider, for example, the quadrilateral sella-condylion-PTM-orbitale. In Figure 8a it is divided into two triangles along the diagonal from sella to PTM. We see a shape change by some $16 \%$ (measured, for instance, by the ratio of distances sella-condylion:condylionPTM) in one triangle, and near-normal form in the other (albeit reduced by about $18 \%$ from normal size). In Figure 8c the same quadrilateral is triangulated along its other diagonal, from condylion to orbitale. Now each triangle shows a diminishment of this diagonal by about $20 \%$ from normal; but in one of the triangles this nearly is the most normal transect, and in the other the least normal! While sella's distance from this diagonal is approximately normal on the average, the mean height of the triangle opposite falls short of normal by nearly $30 \%$. These statements, each true separately, are inconsistent with any single model of real deformation: the analysis is analogous to that in Figure 3-5 rather than Figure 6.

Further contradictory information is supplied about this region of the form in Figure $8 b$; here we "see" a deformation apparently constant over the quadrilateral SER-condylion-PTM-INZ, a deformation which shows distances to be contracted by $30 \%$ of normal in the "northwest-southeast" direction here, but indistinguishable from normal along segments like condylion-SER. These strains in turn are contradicted by those in Figure 8d: the strain -0.31 of triangle condylion-SERPTM, for instance, lies nearly atop the +0.31 pertaining to the triangle basion-sellaSER, and so forth.

Hardly anywhere in this set of landmarks can one find opposing triangles that show similar shape deformities. That triangles PTM-condylion-basion and PTM-condylionsella share a strain tensor implies only that the point condylion, on the temporal bone, can be imagined to be deformed nearly uniformly with the posterior cranial base segment basion-sella: the identity of the two tensors is not a statement about any area. Likewise, the agreement between the tensors describing the changes of triangles nasion-orbitale-ANS and INZ-orbitale-ANS is a statement about the straightness of the "line" nasion-orbitale-INZ. From these finite elements we gain no reliable knowledge of any deformation of areas in the lateral cephalogram.

Any summary analysis from this pile of triangles ought to proceed without reference to deformations. In the absence of any ca- nonical method for summarizing the changes, I proceed as follows. Running up the middle of Figure $8 \mathrm{a}$ is a quadrilateral of landmarks (sella-PTM-INZ-orbitale) which shows nearly normal proportions throughout. A computation using the opposite triangulation, not shown here, confirms that by themselves these four landmarks lie in a normal, albeit quite small, configuration. For all triangles in this quadrilateral, the standard deviations of length-ratios along the directions of largest or smallest mean strain lie in the range of $10-15 \%$. Then the net shape deformation, normative to Apert, is statistically not significant by matched $\mathrm{T}^{2}$ applied to the shape coordinates (Bookstein, 1984b, 1986) or by the approximate t-test of Bookstein (1984a), while the size component of difference is highly significant.

Now no other landmarks appear to participate in this uniform $20 \%$ shortfall of scale. However, there is another near-invariance of normal shape-the cranial base triangle, Fig-
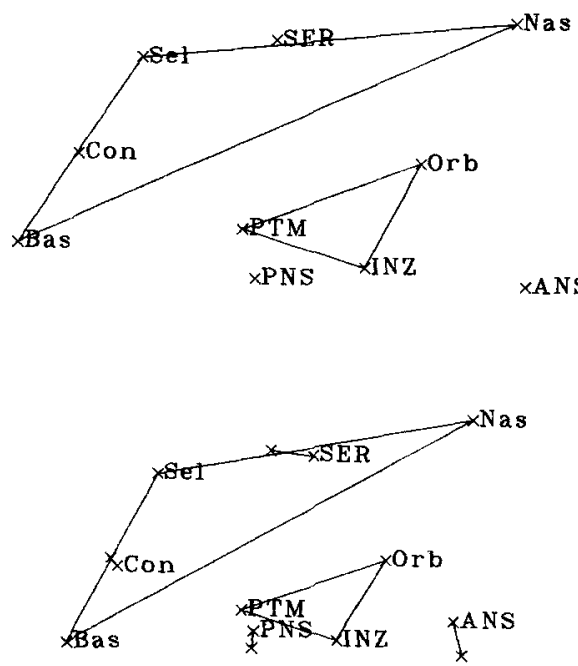

Fig. 9. The author's preferred description of the Apert deformity in terms of these ten landmarks, superimposed upon the mean positions of the landmarks from Figure 7. Both the cranial base (together with the lateral landmark condylion) and the lateral maxilla appear to change scale (by different fractions) without deformation, but the spatial relationship between them is altered. The sphenoidal artifact SER and both palatal landmarks seem translated with respect to these structures. In the lower panel, each of these three outlying landmarks is connected by a dash to the position imputed to it by the uniform scale change of one of these triangles. 
ure 8d, referred to alreadly. (This uniformity extends to the point condylion here and also to the point FMN, the frontomaxillonasal suture: cf. Grayson et al., 1985.) There is a relative rotation of some $5^{\circ}$ between these two forms, together with a discrepancy in their scale changes.

With respect to either of these apparently "fixed" forms, the point SER appears to be considerably displaced. The Apert SER is found, on average, about halfway between sella and nasion, instead of the normal $33 \%$ of the way along that segment, and also slightly below its usual position. Grayson et al. (1985) accept this aspect of the description as real, i.e., as describing actual displacements (not deformations) of the ala sphenoidale forward and the body downward owing to hydrodynamic pressure generated by brain growth within the synostosed calvarium. The body of the sphenoid has presumably deformed in response, but we have no information permitting us to calibrate this deformation.

The remaining two landmarks, ANS and PNS, are substantially displaced from their normal positions with respect to either "fixed" structure. The greatest shortfall involving ANS appears to be in its displacement from orbitale, as mentioned above, and that for PNS appears to be its separation from a point just forward of PTM. The fractions of shortfall from normal are both $40 \%,{ }^{3}$ and the directions make opposite angles with the palatal plane. But both of these distances are severely foreshortened in lateral projection, and no other landmarks are available against which we might check the assertion that the apparent "deformation" of these maxillas is real. In my view, the configuration would best be summarized instead as a relative motion of each palatal landmark with respect to higher midline structure not located here.

For the available data there results the summary diagram of Figure 9. The finiteelement analysis has found two landmark triangles typically normal in shape, but abnormal in size and in relative orientation, and three landmarks further out-of-position

\footnotetext{
${ }^{3}$ The distance of the mean location of PTM from the mean location of PNS (Fig. 7) is reduced in the Apert sample by some $60 \%$ of the normative distance. But the anteroposterior relation between PTM and PNS in the Apert case shows much greater variation than in the Ann Arbor sample, so that the comparison of mean locations itself foreshortens the actual mean distanceratio.
}

with respect to these two structures. A finiteelement analysis deals with shape change as deformation, but this report does not refer to any nontrivial deformations anywhere. It is likely that the reorientation and adjustment of scale discrepancy between the two structures of approximately normal shape-the cranial base triangle basion-sella-nasion and the lateral maxillary triangle-is managed by a deformation of the anonymous regions of calvarium in between them, the regions where there are no data. But there is no evidence regarding the nature of this deformation. (Is sella "misplaced"? How is the discrepancy between scale changes-12\% in the cranial base, $18 \%$ in the maxillabridged?) I consider it unwise to speculate on its geometrical nature. The necessary evidence would instead be histological, beyond the scope of the landmark coordinates available for this analysis.

\section{CONCLUDING REMARKS}

Figure $8 \mathrm{a}-\mathrm{c}$ are all consistent with the available data-the locations of ten landmarks from cephalograms of Apert cases and of normal children-but are grossly inconsistent with each other. The inconsistencies do not have the appearance of "measurement error" that might be corrected by averages over adjacent regions; instead they express a spatial heterogeneity that is irreducible at the level of detail afforded by these data. The data unambiguously detect mean differences of Aperts from normals for nearly all size and shape measures, but do not specify any particular deformation that might best interpret this deformity. The summary which I prefer, Figure 9, finds no "deformation" at all, but instead reports size change at different rates in different regions, together with vectors of relative displacement involving both isolated points and structures of normal shape. Of course, Figure 9 is neither more nor less consistent with the landmark data than any of the panels of Figure 8. Rather, the units it deals with as explanatory entities-the cranial base, the lateral maxilla, and the palate-are the sort of entities to which functional cranial explanations often apply. Mine is, in short, a satisfactorily simple explanation consistent with the data. This is the most that can be required of any cephaiometric landmark analysis. For Figure 6, that simplest explanation is itself a nearly uniform deformation asserting that the form, in effect, does what the variables do. For 
the Apert data, no such simple deformation suits the data; but a complex deformation is a more complicated description than that of Figure 9.

I am not claiming that this is an adequate analysis of the calvarial aspects of Apert syndrome. There are only ten landmarks here; we should have more. Evidence of true deformation, such as in the cranial base, might be supplied by a close study of the curving of form between the landmarks used here, just as in this example we used the position of condylion, however far lateral, as a check on the observation of shape invariance for the superoposterior structures. (But there is at present no consensus among morphometricians about an appropriate statistical method for this study.) These landmarks were located only on X-ray film: the third spatial coordinate is missing, the one automatically obliterated in lateral cephalometrics. Elsewhere in this symposium there are reports of three-dimensional computer graphics for shape inspection which, extended somewhat, may lead to satisfactory three-dimensional landmark data bases. Because Apert syndrome is in part a growth deficiency (that is, the normal form grows in ways prohibited for the Apert calvarium), the description of the deformity should itself vary with age. This latter aspect of the syndrome is one of the topics of Richtsmeier's paper elsewhere in this symposium.

But the fallacies underlying finite-element interpretations in cephalometrics are independent of other problems peculiar to these particular data. One cannot escape the purely biometric paradox: finite elements quite effectively describe the algebra of alternate measurement schemes for landmark data even when they have nothing to do with the processes, deformations or otherwise, that are moving the landmarks around. The biometric analysis of any landmark data set is essentially unique: it is the same for all complete finite-element schemes, but also for all full-information systems of displacement vectors and all competent collections of distances and angles in sufficient variety. Although finite elements underlie this common biometrical substrate, the findings may be reported as true deformations of biologically real finite elements only when the data support such an interpretation with as much redundancy as in the example of Figure 6. In the application to cephalometrics, what the finite-element method describes well is the algebra of possibly helpful variables. It is consistent with excellence of statistics-this is, of course, no mean accomplishment--but it should not claim empirical superiority over statistically equivalent scalar or vector interpretations of greater biological insight.

\section{ACKNOWLEDGMENTS}

This essay, like the others in my series on foundations of morphometrics, was stimulated by conversations with Richard Skalak of Columbia University. I have benefited also from discussions with Gautam Dasgupta of Columbia, Colin Goodall of Princeton, and Paul Sampson of the University of Washington. The writing of this argument was partially supported by N.I.D.R. grants DE- 05410 and GM-37251 to F.L. Bookstein and DE03610 to R.E. Moyers. The gathering of the Apert data was underwritten by grant $\mathrm{DE}$. 03568 to J.G. McCarthy.

\section{LITERATURE CITED}

Bookstein, FL (1978) The Measurement of Biological Shape and Shape Change. Lecture Notes in Biomathematics, v. 24. New York: Springer-Verlag.

Bookstein, FL (1982) On the cephalometrics of skeletal change. Am. J. Orthod. 82:177-198.

Bookstein, FL (1983) The geometry of craniofacial growth invariants. Am. J. Orthod. 83:221-234.

Bookstein, FL (1984a) A statistical method for biological shape comparisons. J. Theor. Biol. 107:475-520.

Bookstein, FL (1984b) Tensor biometrics for changes in cranial shape. Ann. Hum. Biol. 11:413-437.

Bookstein, FL (1985) Transformations of quadrilaterals, tensor fields, and morphogenesis. In PL Antonelli (ed): Mathematical Essays on Growth and the Emergence of Form. Edmonton: University of Alberta Press, pp. 221-265.

Bookstein, FL (1986) Size and shape spaces for landmark data in two dimensions. Stat. Sci. 1:181-242.

Bookstein, FL, and Sampson, PD. Statistical models for geometric components of shape change. J. Am. Stat. Assoc., submitted for publication.

Bookstein, FL, Chernoff, B, Elder, R, Humphries, J, Smith, G, and Strauss, R (1985) Morphometrics in Evolutionary Biology. The Geometry of Size and Shape Change, with Examples from Fishes. Philadelphia: Academy of Natural Sciences of Philadelphia.

Cheverud, JM, Lewis, J, Bachrach, W, and Lew, W (1983) The measurement of form and variation in form: An application of three-dimensional quantitative morphology by finite-element methods. Am. J. Phys. Anthropol. 62:151-165.

Goodall, CR (1983) The Statistical Analysis of Growth in Two Dimensions. Doctoral dissertation, Department of Statistics. Cambridge, MA: Harvard University.

Grayson, B, Weintraub, N, Bookstein, FL, and McCarthy, J (1985) A comparative cephalometric study of the cranial base in craniofacial syndromes. Cleft PaIate J. 22:75-87.

Moss, ML, Skalak, R, Patel, H, Sen, K, Moss-Salentijn, L, Shinozuka, M, and Vilmann, H (1985) Finite element method modeling of craniofacial growth. Am. J. 
Orthod. 87:453-472.

Patel, HC (1983) Growth Analysis by Non-Linear Continuum Theory. Doctoral dissertation. New York: Columbia University.

Riolo, ML, Moyers, R, McNamara, J, and Hunter W
(1974) An Atlas of Craniofacial Growth. Monograph No. 2, Craniofacial Growth Series. Ann Arbor: University of Michigan, Center for Human Growth and Development. 